\title{
Process Control of a Dropwise Additive Manufacturing System for Pharmaceuticals using Polynomial Chaos Expansion Based Surrogate Model
}

Elçin Içtena*, Zoltan K. Nagy ${ }^{\mathrm{a}}$, Gintaras V. Reklaitis ${ }^{\mathrm{a}}$

${ }^{a}$ School of Chemical Engineering, Purdue University, West Lafayette, IN, USA

*Corresponding Author: +17654047190, eicten@purdue.edu

\begin{abstract}
The paper presents a dropwise additive manufacturing process for pharmaceutical products (DAMPP) as an alternative to conventional methods. This mini manufacturing process for the production of personalized pharmaceutical products utilizes drop-on-demand (DoD) printing technology for the deposition of active pharmaceutical ingredient (API) onto edible substrates. Here we present a process control framework for DAMPP, including on-line monitoring, automation and closed loop control, in order to produce individual dosage forms with the desired critical quality attributes, including formulation composition, drop size, deposit morphology and dissolution performance. In order to achieve desired product morphology, a surrogate model based on polynomial chaos expansion is developed to relate the critical process parameters to deposit morphology using dissolution data of the active pharmaceutical ingredient. The proposed process control strategy can effectively mitigate variations in the dissolution profiles due to variable dosage amounts and enable the application of the DoD system for the production of individualized dosage regimens.
\end{abstract}

Keywords: process control, supervisory control, polynomial chaos expansion, surrogate modelling, pharmaceutical process, drop on demand 


\section{Introduction}

In recent years, the US Food and Drug Administration (FDA) introduced the Quality by Design (QbD) approach and Process Analytical Technology (PAT) guidance to encourage innovation and efficiency in pharmaceutical development, manufacturing and quality assurance (Food and Drug Administration CDER, 2004). As part of this renewed emphasis on improvement of manufacturing, the pharmaceutical industry has begun to shift towards continuous processing and to develop more efficient production systems with more intensive use of on-line measurement and sensing, real time quality control and process control tools (Gernaey et al., 2012; Troup and Georgakis, 2012). Improved production processes offer the potential for reduced production costs, faster product release, reduced variability, increased flexibility and efficiency, and improved product quality (Stephanopoulos and Reklaitis, 2011).

Under the US National Science Foundation supported Engineering Research Center for Structured Organic Particulate Systems (NSF ERC-SOPS), a dropwise additive manufacturing process for solid oral drug production has been developed. The process utilizes the drop-ondemand (DoD) printing technology for predictable and highly controllable deposition of active pharmaceutical ingredients (API) onto an edible substrate, such as a polymeric film or placebo tablet, using a semi-continuous operation suitable for low volume production of personalized dosage forms (Hirshfield et al., 2014). Using DoD, different formulations with a wide range of properties can be deposited, including solvent-based and melt-based systems.

The main advantages of using DoD technology for drug printing are the ability to produce small droplets of controlled sizes and to deposit these drops with high placement accuracy (de Gans et al., 2004). The advantages of liquid processing and reproducible production of small droplets create an opportunity in the production of low dose, high potency drugs. By changing the drop 
size and the composition of the formulation, the dosage amount and delivery characteristics can be adjusted according to the patient's needs thereby enabling the process to produce individualized dosage products.

As with any process for producing a drug product, the desired critical quality attributes (CQA), which are its essential physical, chemical and biological characteristics, must be within the appropriate limits defining the desired product quality (Food and Drug Administration CDER, 2008). In order to achieve the desired CQA's, i.e. dosage amount, product morphology and dissolution performance, the critical process parameters (CPP) should be controlled. For this mini-manufacturing system, the CPP's, whose variability has an impact on the CQA's, are the drop size, product and process temperatures. Implementation of a supervisory control system on the process, including on-line monitoring, automation and closed-loop control, is essential for producing individual dosage forms with the desired critical quality attributes. This paper presents such a system, which assures precise control of formulation composition, drop size, deposit morphology and drug dissolution. In order to achieve proper product morphology, a data driven approach based on polynomial chaos expansion (PCE) is used to relate the critical process parameters to deposit morphology using dissolution data of the active pharmaceutical ingredient. The dissolution testing is a standardized off-line laboratory procedure widely used as indicator of bioavailability. The PCE-based surrogate model is then used in an optimal control framework to determine the required temperature profile to achieve a desired bioavailability. In pharmaceutical manufacture, the preferred product quality targets are those closer to reflecting the performance of the product in the patient, such as dissolution, than are traditional quality metrics, such as composition. As noted in recent FDA publications (Lee et al., 2015), the ultimate goal is real time release of product, that is, by passing of the traditional laboratory-based quality control step, 
such as dissolution testing, through use of measurement and advanced control methods during manufacture so as to allow product to be ready for release to the market immediately upon manufacture. The process control strategy reported in this paper is novel not only in providing effective control of the drop on demand manufacturing process but also is one of the first efforts in the literature demonstrating elements of model-based real time release (MBRTR) and the concept of quality-by-control $(\mathrm{QbC})$, whereby product performance and consistent quality are achieved by the design of suitable control strategies.

\section{Process Control Strategy}

The dropwise additive manufacturing system consists of a precision positive displacement pump, xy-staging, a hot air based heating system, on-line imaging and sensing, and temperature, pump and stage controllers, as shown in Fig. 1. The interested reader is invited to refer to Hirshfield et al. (2014) and Içten et al. (2015) for more detailed description of the dropwise additive manufacturing system for solvent-based and melt-based pharmaceutical products, respectively. The above mentioned publications discuss the rationale for developing the drop-on-demand process for pharmaceutical applications and implementation of the DoD system for different pharmaceutical formulations. The Liquid Dispensing Technology (LDT) patented by GSK (Clarke et al., 2012) is used to produce pharmaceutical products by solvent deposition and operates in open loop where the drop sizes are monitored and off-spec tablets are simply rejected. The LDT has no supervisory control implemented for morphology; and quality control on dissolution is handled in a classical sampled fashion.

This paper presents a supervisory control strategy to achieve the critical quality attributes of solid oral dosages produced with the DoD system. The supervisory control strategy for the dropwise additive manufacturing process is shown in Fig. 2. The supervisory control strategy consists of 
two main parts: an automated low-level control system and a surrogate model based supervisory control layer, which are described in Sections 2.1 and 2.2, respectively. The low level control system provides effective control of the drop on demand manufacturing process, whereas the surrogate model based supervisory control layer demonstrates the indirect control of drug dissolution online and enables model-based real time release of solid oral dosages.

\subsection{Low-level Control System}

Automation of the on-line monitoring and control systems is implemented using the LabVIEW (National Instruments) environment. The automation logic controls the pump, camera and xystaging, simultaneously. An image of each drop is taken after it is ejected from the nozzle. The dosage amount of each drop is monitored using the corresponding drop volume calculated using real-time image analysis with arbitrary rotational symmetric shape model (Hugli and Gonzalez, 2000). By changing the pump and nozzle parameters, different drop sizes can be produced, which allows adjustment of the dosage amount for patients with different therapeutic requirements. The xy-staging and synchronization logic allows precise drop positioning on the substrate while printing and enables layering of different drugs, thus offering the flexibility of producing combination dosages. For more details on the real time process management strategy, including automation, online monitoring and exceptional events management, the interested reader is referred to Hirshfield et al. (2015), where the drop deposition, image processing and synchronized xy-staging operation is described.

Using this process, different drug formulations including polymer-API systems, i.e. melts, and solvent-polymer-API systems can be produced. The printability and reproducibility of melt formulations depend on the process temperature, which is maintained above the melting temperature and within the desired operating limits in order to produce hot-melts of API and a 
carrier such as a polymer or surfactant. Therefore temperature control is implemented on reservoir, pump, tubing and nozzle using heating tape, built-in pump heater and air heating system, respectively.

The product morphology depends on the formulation composition, on the selection of the substrate and on the CPP's, i.e. product temperature and drop size. The selection of the polymer used in the formulation can change the morphology by promoting or inhibiting crystallization of the drug (Trasi and Taylor, 2012). The surface properties of the substrate onto which the drops are deposited can also have an effect on product morphology (Hsu et al., 2013). In bulk crystallization processes, crystallization temperature is used to control the crystal properties including size distribution, shape and morphology (Fujiwara et al., 2005; Nagy and Braatz, 2012). In the DAMPP process, the product temperature corresponds to the crystallization temperature after the drop is deposited on the substrate. By manipulating the substrate temperature profile using varying temperature gradients, the drop solidification process can be controlled. The drop size also affects the drop solidification process by changing the heat transfer dynamics. Since the crystallization temperature profile has a strong effect on product solid state and morphology, precise control of the drop solidification process occurring on the substrate is critical (Icten et al., 2014). The temperature of the deposited drops is controlled via the Peltier devices placed on the xy-staging underneath the substrate. The Peltier temperature control is implemented through a PID loop. Programmed temperature gradients including step changes, ramping heating or cooling, cycling or any combination of these temperature profiles can be applied to the drug deposits using the LabVIEW based automation of the substrate temperature control system. 


\subsection{Surrogate Model-based Supervisory Control System}

A surrogate model-based supervisory control system is implemented on top of the low-level control system to ensure that the drug products have the desired product morphology regardless of their dosage amount, i.e. drop size. This can be achieved by monitoring the drop size via the online imaging system and manipulating the temperature profile applied on the substrate for the measured drop size to ensure consistent quality attributes. The crystallization temperature profile has a strong effect on product solid state and morphology, which influence the dissolution performance of the drug.

In the pharmaceutical industry, drug dissolution testing is performed as a standard requirement to provide critical in vitro drug release information for quality control purposes of solid oral dosage forms. The effectiveness of solid oral dosage forms relies on the drug dissolving in the fluids of the gastrointestinal tract prior to absorption into the systemic circulation. The rate of dissolution of a dosage form therefore influences the amount of drug available to the body, i.e. its bioavailability. Since inadequacies in bioavailability can result in ineffective or excessive treatment, precise control of the drop solidification process occurring on the substrate is critical.

In order to optimize the temperature profile applied on the substrate, we use a data driven modelling approach which relates the temperature profiles to the dissolution properties of the drug as a measure of product quality. With this approach, consistent dissolution profile can be achieved for drug products containing different dosage amounts.

In Section 3, the effect of critical process parameters on product quality is investigated. This knowledge is used to build a surrogate model-based control system, which is described in Section 4, and to optimize the temperature profiles as outlined in Section 5. 


\section{Effect of Critical Process Parameters on Product Quality}

In order to study the effects of critical process parameters on the product morphology, dosage forms of melts were produced using two different drop sizes, and applying different substrate temperature profiles. The model formulation of melts is a mixture of naproxen and polyethylene glycol 3350 (PEG 3350) with a weight composition of (15:85), a composition at which they form a eutectic (Zhu et al., 2013). The formulation was co-melted at $65^{\circ} \mathrm{C}$ and the temperature of the system was controlled at $70{ }^{\circ} \mathrm{C}$ throughout the process. Polymeric films prepared with hydroxypropyl methylcellulose (HPMC) (E50) and PEG 400 were used as the substrate. Dosage forms containing $15 \mathrm{mg}$ of API were produced either with large drops of size $23.4 \mathrm{mg}$ with a standard deviation of $1.4 \mathrm{mg}$ or small drops of size $19.4 \mathrm{mg}$ with a standard deviation of $0.3 \mathrm{mg}$. The average drop sizes and the corresponding standard deviations are calculated based on 12 replicates of dosage forms printed.

After the deposition, different cooling temperature profiles are applied to the substrates containing the drops. The molten deposits are cooled down to $30{ }^{\circ} \mathrm{C}$ using one of the three temperature profiles shown in Fig. 3. Constant temperature profile is achieved by printing the drops onto films maintained at $30{ }^{\circ} \mathrm{C}$ and by controlling its temperature at $30{ }^{\circ} \mathrm{C}$ until the drops are solidified. The other dosage forms were printed onto films maintained at $60{ }^{\circ} \mathrm{C}$ and cooled down to $30{ }^{\circ} \mathrm{C}$ using either a fast cooling rate of $10{ }^{\circ} \mathrm{C} / \mathrm{min}$, or a slow cooling rate of $1{ }^{\circ} \mathrm{C} / \mathrm{min}$. The aim is to investigate the effect of different cooling profiles on the product morphology. The points marked on the fast and slow cooling temperature profile curves represent the induction times for crystal formation. Crystallization of the dosage forms is recorded under different cooling temperature profiles using a Zeiss Axio Imager A2m polarized light microscope equipped with a Linkam THMS 600 hot stage. The captured images are analyzed visually to 
determine the time and temperature corresponding to the first nucleus formation. The measurements are taken in two replicates to determine the mean induction temperatures and times. The differences in the induction points show that applying different cooling profiles changes the crystallization behavior. With fast cooling, the induction occurs at $36.6{ }^{\circ} \mathrm{C}$ in 4.3 min, while with slow cooling, the induction temperature is $45.6^{\circ} \mathrm{C}$ occurring in $16.4 \mathrm{~min}$. Since the differences in induction temperatures and times showed small variations between the replicates, additional replicates were not run given the trends observed.

With the model formulations used in this study, the amorphous form of naproxen is not observed in the presence of PEG, which promotes crystallization of naproxen (Zhu et al., 2013). However morphological differences are observed between the dosage forms using optical microscopy when different temperature cooling profiles are applied to the deposits. Fast cooling creates more nucleation sites during crystallization, thus higher surface area of crystals.

Dissolution testing was performed to show the effect of the drop size and the substrate temperature profile on the dissolution behavior of the drug. In Fig. 4.a, the dissolution profiles of the dosage forms printed with small drop size and solidified using fast and slow cooling rates are shown. In Fig. 4.b, the dissolution profiles of the dosage forms printed with large drop size and solidified using fast and slow cooling rates are shown. Dissolution experiments are performed in three replicates, which is the normal practice in the pharmaceutical technology literature, and the estimated standard deviation of the replicates are shown in Fig. 4 in terms of the error bars. Of course, additional replicates would be required to allow rigorous statistical testing of differences. Here we are simply interested in illustrating trends. For the dosage forms printed either with small or large drop sizes, faster dissolution is observed when the fast cooling rate of $10{ }^{\circ} \mathrm{C} / \mathrm{min}$ is 
applied. During fast cooling, creation of more nucleation sites results in higher surface area and therefore in faster dissolution of the dosage forms.

Dissolution profiles of the dosage forms created with two different drop sizes and solidified at different temperature profiles are depicted in Fig. 5. Dissolution experiments are performed in three replicates and the standard deviation of the replicates are represented as the error bars. When the dosage forms created with small or large drops are deposited onto the substrate at a constant temperature of $30^{\circ} \mathrm{C}$, high variation of the dissolution profile is observed within the same drop size, as well as between the small and large drops. When the substrate temperature is constant, the cooling profile within the droplets is significantly influenced by their volume, yielding to large variations in the crystallization conditions. This variation can be seen in terms of the error bars in Fig. 5.a. In the case of solidification of the dosage forms by applying a fast cooling rate of $10^{\circ} \mathrm{C} / \mathrm{min}$, the differences within the same drop size decrease, which is reflected in the reduced error bars, Fig. 5.b. When the dosage forms are solidified by applying a slow cooling rate of $1{ }^{\circ} \mathrm{C} / \mathrm{min}$, the differences both within the same drop size and between different drop sizes decrease, Fig. 5.c. Applying slow cooling profile, decreases the differences in the temperature variations due to the drop size by allowing more time for more uniform heat transfer also decreasing the differences due to the spatial distribution of the temperature within the deposit on the substrate.

Although the amorphous form of naproxen is not exhibited with this formulation, it can be produced with alternative choices of operating conditions and polymers, which inhibit crystallization. Amorphous drug forms can offer improved dissolution performance thus higher bioavailability. However, since the amorphous form is a metastable state of the API, precise temperature control and design of crystallization temperature profile can even be more important 
in tailoring the in vitro or in vivo dissolution profile of the resulting formulation, than in the case of crystalline API formulations.

\section{Polynomial Chaos Expansion Based Surrogate Model Development}

Due to the nonlinear behavior of the solidification and crystallization processes occurring within the melt drops and due to the presence of disturbances, the typical linear data driven modeling approaches may be challenged in relating the crystallization temperature profiles to the dissolution profile with acceptable accuracy. A methodology based on nonlinear data driven modelling approaches such as artificial neural networks (ANN) or polynomial chaos expansions (PCE) can be used to develop a surrogate model describing this system. A surrogate model can be thought of as a "regression" to a set of data, where the data is a set of input-output pairings obtained by evaluating a black-box model of the complex system (Caballero and Grossmann, 2008; Eason and Cremaschi, 2014). Maintaining fidelity while being computationally economical are the main aspects to consider while choosing a surrogate model for a certain application (Biegler et al., 2014).

ANNs are mathematical models consisting of interconnected simple processing units, known as neurons, represented in input-output layers along with hidden layer(s) (Dua, 2011).The parameters of a network include weights and biases, and the operation performed at each neuron. The problem of fitting these parameters is known as training the network. One disadvantage of ANNs is the need for selecting the number of neurons, which must be sufficiently high to capture functional behavior but not so high as to cause overfitting (Eason and Cremaschi, 2014). In the case of a limited number of experimental data, capturing functional behavior can be a challenge. Another disadvantage of ANNs is that there are no mechanisms for taking into account the nature of the distribution functions of the uncertainties in the data. 
Polynomial chaos is a type of spectral method with useful properties that can be exploited for the computations of surrogate model generation and parameter determination (Kim et al., 2013). Polynomial chaos expansion was introduced by (Wiener, 1938) for turbulence modeling (Wiener, 1938) and it became popular only in the last few decades after the surge of fast computers with parallel computing ability (Kim et al., 2013). The implementation of PCEs in terms of Hermite polynomials for linear elastic problems (Ghanem and Spanos, 1991) started broadening the application area of this method. More recently, since the discovery of the possible uses of PCE to define the uncertain model as a deterministic model with an extended number of variables, PCE has been applied in a large variety of disciplines (Nagy and Braatz, 2010). Its application areas include computational fluid dynamics (Zhang and Lu, 2004), robust design problems (Ghisu et al., 2011) and chemical processes (Nagy and Braatz, 2010, 2007).

In this work, we use a methodology based on polynomial chaos expansion (PCE) containing orthogonal basis with respect to the Gaussian probability measure to develop a surrogate model (Kim et al., 2013). PCE can be used to replace a nonlinear system with a surrogate model that adequately describes the input to state and input to output behavior (Nagy and Braatz, 2007). PCE offers three main advantages: small number of parameters hence efficiency in using experimental data, ability to incorporate information about parameter uncertainty and an expansion structure which allows convenience incorporation of additional nonlinear teams if the data requires it. If the parameter uncertainties are described in terms of standard normal random variables, the PCE can describe the model output $\psi$ as an expansion of multidimensional Hermite polynomial functions of the uncertain parameters $\theta$ (Nagy and Braatz, 2007). Using the Hermite bases in the PCE, the output can be expressed in terms of the standard random normal variables $\theta_{i}$ using an expansion of order $d$ : 


$$
\psi^{(d)}=\underbrace{a_{o}^{(d)} \Gamma_{o}}_{\text {constant }}+\underbrace{\sum_{i_{1}=1}^{n_{\theta}} a_{i_{1}}^{(d)} \Gamma_{1}\left(\theta_{i_{1}}\right)}_{\text {firstorderterms }}+\underbrace{\sum_{i_{1}=1}^{n_{\theta}} \sum_{i_{2}=1}^{i_{1}} a_{i_{1} i_{2}}^{(d)} \Gamma_{2}\left(\theta_{i_{1}}, \theta_{i_{2}}\right)}_{\text {sec ondorderterms }}+\underbrace{\sum_{i_{1}=1}^{n_{\theta}} \sum_{i_{2}=1}^{i_{1}} \sum_{i_{3}=1}^{i_{2}} a_{i_{1} i_{2}}^{(d)} \Gamma_{3}\left(\theta_{i_{1}}, \theta_{i_{2}}, \theta_{i_{3}}\right)}_{\text {thirdorderterms }}+\ldots
$$

where $n_{\theta}$ is the number of parameters and $a_{i_{1}}^{(d)}, a_{i_{1} i_{2}}^{(d)}, a_{i_{1} i_{2} i_{3}}^{(d)}, \ldots$ are deterministic coefficients in $\mathrm{R}$ to be estimated (Nagy and Braatz, 2007). The multidimensional Hermite polynomials of degree $m=i_{1}, i_{2}, \ldots, i_{n_{\theta}}, \Gamma_{m}\left(\theta_{i_{1}}, \ldots, \theta_{m}\right)$ are

$$
\Gamma_{m}\left(\theta_{i_{1}}, \ldots, \theta_{m}\right)=(-1)^{m} e^{1 / 2 \theta^{T} \theta} \frac{\partial^{m} e^{-1 / 2 \theta^{T} \theta}}{\partial \theta_{1} \ldots \partial \theta_{m}}
$$

The number of coefficients $N$ in the PCE depends on the number of uncertain parameters and the order of expansion. It can be calculated as

$$
N=1+\frac{n_{\theta} !}{\left(n_{\theta}-1\right) ! 1 !}+\frac{\left(n_{\theta}+1\right) !}{\left(n_{\theta}-1\right) ! 2 !}+\frac{\left(n_{\theta}+2\right) !}{\left(n_{\theta}-1\right) ! 3 !}+\ldots+\frac{\left(n_{\theta}+m-1\right)}{\left(n_{\theta}-1\right) ! m !}
$$

For most engineering applications, the use of third or fourth order expansion is sufficient. However, the determination of the most appropriate number of terms to be used in the expansion needs to be made for each application. For instance Nagy and Braatz (2007) reported that a second order model was sufficient in the case of a batch crystallization process application and thus that the use of third order PCE model was not required. The use of high order expansion increase the number of coefficients required for the model development. In the case of limited number of experimental data available, lower order PCE models are preferred.

The polynomial chaos expansion is convergent in the mean-square sense (Ghanem and Spanos, 1991). Therefore the coefficients in the PCE $a_{i_{1}}^{(d)}, a_{i_{1} i_{2}}^{(d)}, a_{i_{1} i_{2} i_{3}}^{(d)}, \ldots$ can be calculated using least square minimization by considering sample input-output pairs from the model to achieve the best fit 
between the surrogate PCE model and the experimental data (Nagy and Braatz, 2007). The initial coefficients in the PCE are selected randomly. Since the initial coefficients affect the accuracy of the calculations, the coefficients from the previous solution are used to perform the least square minimization iteratively until there is no significant difference between the predicted outputs. In this manner, the best PCE parameters can be determined. The steps of the surrogate model development using PCE are shown in the flowchart in Fig. 6.

This computationally efficient method is used to generate a data driven input-output model relating temperature control profiles and the dissolution profiles of the dosage forms produced with the dropwise additive manufacturing process. In the pharmaceutical industry, it is common practice to treat the uncertainties in dissolution data as normally distributed (Tsong et al., 2004). Moreover, uncertainties in temperature measurements via thermocouples are generally accepted to have normal distributions. Consequently, we assumed normal distribution on the model input data and therefore used orthogonal Hermite polynomials in the PCE model. However if the uncertainties are not normally distributed, then different orthogonal polynomials with different distribution types can be used in PCE model development, including Jacobi, Legendre, Laguerre and Chebyshev polynomials (Kim et al., 2013).

In Fig. 7, two different representations of the model inputs, i.e. temperature profiles, and the model outputs, i.e. dissolution profiles, are shown. Different PCE models can be developed with different numbers of input data, which are required to describe the system accurately. In Fig. 7-a, (1-1) PCE model representing single input-single output case is shown. For (1-1) PCE model, the temperature control profiles are described with a single input data representing the linear cooling rate applied to the drops. In Fig. 7-b, (5-1) PCE model representing multiple input-single output case is shown. For (5-1) PCE model, the temperature control profiles are represented as the 
temperature values corresponding to discrete times. These time values are selected to ensure distinct discretization between different temperature profiles. In addition to better accuracy, another advantage of the (5-1) PCE model is that more complex temperature trajectories can be included in the hierarchical control scheme, not only linear cooling, enabling better control of the dissolution profile. The output data is calculated as the characteristic time constant $\tau$ corresponding to time to reach $60 \%$ of total dissolution, which would be an approximation of the time constant of the dissolution profile, approximating the dissolution process with first order dynamics. Eq. 4 gives the functional input-output representation for the (1-1) PCE model,

$$
\begin{aligned}
& \tau_{P C E}^{(1-1)}=f_{P C E}^{(1-1)}(R) \\
& \tau_{P C E}^{(1-1)}=a_{0} \Gamma_{0}+\sum_{i=1}^{n_{\theta}} a_{i} \Gamma_{1}\left(\theta_{i}\right), n_{\theta}=1 \\
& R=\Gamma_{1}\left(\theta_{i}\right), \quad i=1
\end{aligned}
$$

where $a_{i}$ are the PCE coefficients, $\Gamma_{1}\left(\theta_{i}\right)$ are the first degree Hermite polynomials, and $R$ is the linear cooling rate applied to the drops. Eq. 5 gives the functional input-output representation for the (5-1) PCE model,

$$
\begin{aligned}
& \tau_{P C E}^{(5-1)}=f_{P C E}^{(5-1)}\left(\left.T_{i}\right|_{i=1,2, \ldots, 5}\right) \\
& \tau_{P C E}^{(5-1)}=a_{0} \Gamma_{0}+\sum_{i=1}^{n_{\theta}} a_{i} \Gamma_{1}\left(\theta_{i}\right), n_{\theta}=5 \\
& T_{i}=\Gamma_{1}\left(\theta_{i}\right), \quad i=1, \ldots, 5
\end{aligned}
$$

where $a_{i}$ are the PCE coefficients, $\Gamma_{1}\left(\theta_{i}\right)$ are the first degree Hermite polynomials, and $T_{n_{\theta}}$ are the discretized temperature values.

As a case study, dosage forms of melts containing $15 \mathrm{mg}$ of API were produced by depositing 5 drops with the average size of $18.6 \mathrm{mg}$ with a standard deviation of $0.6 \mathrm{mg}$. The average drop 
sizes and the corresponding standard deviations are calculated based on 21 replicates of dosage forms. The same model formulation, substrate and process temperatures are used throughout the process as described in Section 3. After the deposition, different controlled cooling profiles, which are shown in Fig. 8, are applied to the dosage forms with 3 replicates at each condition. The corresponding dissolution profiles are used to test a 1st order PCE with different number of input parameters. Single value cooling rates are used as the single input parameters for (1-1) PCE model. Discretized cooling profiles are obtained by using the temperature values corresponding to $0,2,10,30,60$ minutes during cooling and are used as multiple input parameters for (5-1) PCE model. Depending on the number of input parameters, different numbers of coefficients are required to build the model. In this case study, $N=2$ and $N=6$ coefficients are used for the PCE models with single input (1-1) and with multiple input (5-1), respectively. The single input, i.e. cooling rates, and multiple input, i.e. discretized temperature profiles, along with the output data, i.e. the time constants of the dissolution profiles, used in the model development are listed in Table 1.

Next, the PCE models are subjected to leave-one-out cross-validation and resampling to get a better comparison on the prediction capability of the single input (1-1) and 5 input (5-1) PCE models. Here validation data both within and outside the range of training data are used. For both models, the mean square error of estimation (MSEE) and mean square error of prediction (MSEP) are calculated and shown in Table 2. The model fit and prediction capabilities of the (11) PCE model and the (5-1) PCE model are shown in Fig. 9-a and 9-b, respectively. The average residuals of (1-1) and (5-1) models are $15.0 \%$ and $4.4 \%$, respectively. The (5-1) PCE model provides better prediction than the (1-1) PCE model both with lower residuals and with lower MSEE and MSEP values. 


\section{Surrogate Model Based Optimization of Temperature Profiles}

After validation, both of the PCE models, i.e. (1-1) and (5-1) PCE models, are used to determine the optimal temperature profiles needed to reach the desired process outcome, i.e. time constant $\tau$ of the dissolution profile. The predicted temperature profiles using different models are compared qualitatively. The data points which are left out for cross validation are used in the prediction of the temperature profiles.

The optimal control formulation based on the (1-1) PCE model used in the higher level supervisory controller is given by Eq. 6 . The minimum and maximum temperature cooling rates

are $R_{\min }=0.5^{\circ} \mathrm{C} / \mathrm{min}$ and $R_{\max }=15^{\circ} \mathrm{C} / \mathrm{min}$, respectively. The average of the seven cooling rates, shown in Figure 8, is used to initialize the optimization problem.

$$
\begin{array}{ll}
\min _{R}\left(\tau_{\text {desired }}-\tau_{P C E}^{(1-1)}\right)^{2} \\
\text { s.t. } \quad \tau_{P C E}^{(1-1)}=a_{0} \Gamma_{0}+\sum_{i=1}^{n_{\theta}} a_{i} \Gamma_{1}\left(\theta_{i}\right), \quad n_{\theta}=1 \\
\quad R=\Gamma_{1}\left(\theta_{i}\right), \quad i=1 \\
\quad R_{\min } \leq R \leq R_{\max }
\end{array}
$$

The optimal control formulation used in the supervisory control in the case of the (5-1) PCE model is given by Eq. 7. The initial and final temperatures are fixed at $T_{\text {initial }}=60{ }^{\circ} \mathrm{C}$ and $T_{\text {final }}=30^{\circ} \mathrm{C}$, respectively. The average of the seven cooling rates, shown in Figure 8, is discretized at the same time points that are used for the discretization during (5-1) PCE model development. Then this discretized average cooling profile is used to initialize the optimization problem. 


$$
\begin{array}{ll}
\min _{T}\left(\tau_{\text {desired }}-\tau_{P C E}^{(5-1)}\right)^{2} & \\
\text { s.t. } \quad & \tau_{P C E}^{(5-1)}=a_{0} \Gamma_{0}+\sum_{i=1}^{n_{\theta}} a_{i} \Gamma_{1}\left(\theta_{i}\right), \quad n_{\theta}=5 \\
& T=\left[T_{1}, T_{2}, \ldots, T_{5}\right] \\
T_{i} & =\Gamma_{1}\left(\theta_{i}\right), \quad i=1,2, \ldots, 5 \\
& T_{i} \geq T_{i+1}, \quad i=1,2, \ldots, 4 \\
& T_{1}=T_{\text {initial }} \\
T_{5} & =T_{\text {final }}
\end{array}
$$

The optimal temperature profiles are shown in Fig. 10. Using both the (1-1) and (5-1) models, for faster cooling rates of $15{ }^{\circ} \mathrm{C} / \mathrm{min}$ and $10{ }^{\circ} \mathrm{C} / \mathrm{min}$, the predicted temperature profiles to reach the desired dissolution profiles compare well with the actual temperature profiles applied to the dosage forms. However for a cooling rate of $1{ }^{\circ} \mathrm{C} / \mathrm{min}$, the $(5-1)$ model gives a better prediction compared to (1-1) model. The prediction capabilities of the models show that the (5-1) PCE model captures the behavior of the system better. Using discretized temperature profiles can also allow the use of optimized temperature cycles with variable cooling and/or heating rates.

By using more experimental data points, the PCE based surrogate model can be improved to have better model fit and also better prediction. A higher order PCE model can also be developed with more data points. Moreover, using a different pharmaceutical formulation, which would undergo not only morphological but also crystallinity changes depending on the applied control strategies, can result in more diverse dissolution profiles.

A hierarchical control strategy can be implemented on the dropwise additive manufacturing process by monitoring the drop size on-line, and selecting a PCE model from the PCE model family developed for different drop sizes. One such hierarchical control strategy is shown in the flowchart in Fig. 11. Using the proposed hierarchical control strategy, for a measured drop size, 
the temperature profiles can be optimized to reach the desired process outcome, in this case, a desired dissolution profile.

\section{Conclusions}

In this work, a process control strategy for the dropwise additive manufacturing system is reported. Using the imaging system, the drop sizes and therefore the dosage amounts are monitored. Using the proposed substrate temperature control strategy, the crystallization behavior can be tailored and consistent drug morphology can be achieved. A PCE based surrogate model is developed to predict the dissolution profile of the solidified drug deposition given the temperature profile applied on the substrate. Using this model, a hierarchical control system is being implemented by monitoring the drop size on-line and predicting a temperature profile to achieve the desired dissolution profile for the dosage forms created. The process control strategy reported in this paper is novel not only in providing effective control of the drop on demand manufacturing process but also is one of the first efforts in the literature demonstrating elements of model-based real time release and the concept of quality-by-control, whereby product performance and consistent quality are achieved by the design of suitable control strategies.

The reported process control strategy can effectively mitigate variations in the dissolution profiles due to variable dosage amounts, hence enabling the application of the DoD system for the production of individualized dosage regimens for adaptive clinical trials and personalized treatments. The prototype system offers great promise as a tool for advancing personalized medicine by allowing the precise production of convenient solid oral dosages tailored to the patient on site at hospitals, clinics and even pharmacies. 


\section{Acknowledgments}

This work was funded by the National Science Foundation under grant EEC-0540855 through the Engineering Research Center for Structured Organic Particulate Systems. The authors would like to thank Indiana Next Generation Manufacturing Competitiveness Center (IN-MaC) for financial support provided to E.I. The authors are grateful for the advice and counsel of Arun Giridhar on a range of technical implementation issues. The authors would like to thank Golshid Kevyan for performing the dissolution analysis of the dosage forms. 


\section{References}

Biegler, L.T., Lang, Y., Lin, W., 2014. Multi-scale optimization for process systems engineering. Comput. Chem. Eng. 60, 17-30. doi:10.1016/j.compchemeng.2013.07.009

Caballero, J.A., Grossmann, I.E., 2008. An Algorithm for the Use of Surrogate Models in Modular Flowsheet Optimization 54, 2633-2650. doi:10.1002/aic

Clarke, A.J., Doughty, D.G., Fiesser, F.H., Wagner, D.S., 2012. Apparatus for producing a pharmaceutical product. Patent US 8252234.

deGans, B.-J., Duineveld, P.C., Schubert, U.S., 2004. Inkjet Printing of Polymers: State of the Art and Future Developments. Adv. Mater. 16, 203-213. doi:10.1002/adma.200300385

Dua, V., 2011. An Artificial Neural Network approximation based decomposition approach for parameter estimation of system of ordinary differential equations. Comput. Chem. Eng. 35, 545-553. doi:10.1016/j.compchemeng.2010.06.005

Eason, J., Cremaschi, S., 2014. Adaptive sequential sampling for surrogate model generation with artificial neural networks. Comput. Chem. Eng. 68, 220-232. doi:10.1016/j.compchemeng.2014.05.021

Food and Drug Administration CDER, 2008. Q8 (R1) Pharmaceutical Development Revision 1. Silver Spring, Maryland.

Food and Drug Administration CDER, 2004. Guidance for Industry PAT - A Framework for Innovative Pharmaceutical. Silver Spring, Maryland.

Fujiwara, M., Nagy, Z.K., Chew, J.W., Braatz, R.D., 2005. First-principles and direct design approaches for the control of pharmaceutical crystallization. J. Process Control 15, 493504. doi:10.1016/j.jprocont.2004.08.003

Gernaey, K. V., Cervera-Padrell, A.E., Woodley, J.M., 2012. A perspective on PSE in pharmaceutical process development and innovation. Comput. Chem. Eng. 42, 15-29. doi:10.1016/j.compchemeng.2012.02.022

Ghanem, R.G., Spanos, P.D., 1991. Stochastic Finite Elements: A Spectral Approach. SpringerVerlag, New York.

Ghisu, T., Parks, G.T., Jarrett, J.P., Clarkson, P.J., 2011. Robust Design Optimization of Gas Turbine Compression Systems. J. Propuls. Power 27, 282-295. doi:10.2514/1.48965

Hirshfield, L., Giridhar, A., Taylor, L.S., Harris, M.T., Reklaitis, G. V, 2014. Dropwise additive manufacturing of pharmaceutical products for solvent-based dosage forms. J. Pharm. Sci. 103, 496-506. doi:10.1002/jps.23803 
Hirshfield, L., Içten, E., Giridhar, A., Nagy, Z.K., Reklaitis, G. V., 2015. Real-Time Process Management Strategy for Dropwise Additive Manufacturing of Pharmaceutical Products. J. Pharm. Innov. doi:10.1007/s12247-015-9218-5

Hsu, H., Toth, S.J., Simpson, G.J., Taylor, L.S., Harris, M.T., 2013. Effect of Substrates on Naproxen-Polyvinylpyrrolidone Solid Dispersions Formed via the Drop Printing Technique 102, 638-648. doi:10.1002/jps

Hugli, H., Gonzalez, J., 2000. Drop volume measurements by vision. Imaging 2000, SPIE Electron. Imaging Conf. San Diego 3966, 60-66.

Içten, E., Giridhar, A., Taylor, L.S., Nagy, Z.K., Reklaitis, G. V, 2015. Dropwise Additive Manufacturing of Pharmaceutical Products for Melt-Based Dosage Forms. J. Pharm. Sci. 104, 1641-1649. doi:10.1002/jps.24367

Icten, E., Nagy, Z.K., Reklaitis, G. V., 2014. Supervisory Control of a Drop on Demand Minimanufacturing System for Pharmaceuticals. Proc. 24th Eur. Symp. Comput. Aided Process Eng., Computer Aided Chemical Engineering 33, 535-540. doi:10.1016/B978-0-44463456-6.50090-9

Kim, K., Shen, D.E., Nagy, Z.K., Braatz, R.D., 2013. Wiener's Polynomial Chaos for the Analysis and Control of Nonlinear Dynamical Systems with Probabilistic Uncertainties. IEEE Control Syst. Mag. 58-67.

Lee, S.L., O'Connor, T.F., Yang, X.,Cruz, C.N., Chatterjee, S., Madurawe, R.D., Moore, C.M.V., Yu, L.X., Woodcock, J., 2015. Modernizing Pharmaceutical Manufacturing: form Batch to Continuous Production. J. Pharm. Innov. doi: 10.1007/s12247-015-9215-8.

Nagy, Z.K., Braatz, R.D., 2012. Advances and new directions in crystallization control. Annu. Rev. Chem. Biomol. Eng. 3, 55-75. doi:10.1146/annurev-chembioeng-062011-081043

Nagy, Z.K., Braatz, R.D., 2010. Distributional uncertainty analysis using polynomial chaos expansions. 2010 IEEE Int. Symp. Comput. Control Syst. Des. 1103-1108. doi:10.1109/CACSD.2010.5612662

Nagy, Z.K., Braatz, R.D., 2007. Distributional uncertainty analysis using power series and polynomial chaos expansions. J. Process Control 17, 229-240. doi:10.1016/j.jprocont.2006.10.008

Stephanopoulos, G., Reklaitis, G. V., 2011. Process systems engineering: From Solvay to modern bio- and nanotechnology. Chem. Eng. Sci. 66, 4272-4306.

doi:10.1016/j.ces.2011.05.049

Trasi, N.S., Taylor, L.S., 2012. Effect of polymers on nucleation and crystal growth of amorphous acetaminophen. CrystEngComm 14, 5188. doi:10.1039/c2ce25374g 
Troup, G.M., Georgakis, C., 2012. Process systems engineering tools in the pharmaceutical industry. Comput. Chem. Eng. 1-15. doi:10.1016/j.compchemeng.2012.06.014

Tsong, Y., Shen, M., Shah, V., 2004. Three-stage sequential statistical dissolution testing rules. J. Biopharm. Stat. 14, 757-779.

Wiener, N., 1938. The Homogeneous Chaos. Am. J. Math. 60, 897-936.

Zhang, D., Lu, Z., 2004. An efficient, high-order perturbation approach for flow in random porous media via Karhunen-Loève and polynomial expansions. J. Comput. Phys. 194, 773794. doi:10.1016/j.jcp.2003.09.015

Zhu, Q., Toth, S.J., Simpson, G.J., Hsu, H.-Y., Taylor, L.S., Harris, M.T., 2013. Crystallization and dissolution behavior of naproxen/polyethylene glycol solid dispersions. J. Phys. Chem. B 117, 1494-500. doi:10.1021/jp3106716 
Table 1 Single Input, Multiple Input and Single Output Parameters for PCE based Model Development

\begin{tabular}{|c|c|c|}
\hline Cooling Rates & Discretized Temperature Profiles & Dissolution Time Constants $\tau$ \\
\hline $15^{\circ} \mathrm{C} / \mathrm{min}$ & {$[6030303030]$} & $8 \mathrm{~min}$ \\
\hline $10^{\circ} \mathrm{C} / \mathrm{min}$ & {$[6040303030]$} & $8.8 \mathrm{~min}$ \\
\hline $3^{\circ} \mathrm{C} / \mathrm{min}$ & {$[6054303030]$} & $9.6 \mathrm{~min}$ \\
\hline $1.5^{\circ} \mathrm{C} / \mathrm{min}$ & {$[6057453030]$} & $10.2 \mathrm{~min}$ \\
\hline $1^{\circ} \mathrm{C} / \mathrm{min}$ & {$[6058503030]$} & $10.7 \mathrm{~min}$ \\
\hline $0.75^{\circ} \mathrm{C} / \mathrm{min}$ & {$[6058.552 .537 .530]$} & $11.5 \mathrm{~min}$ \\
\hline $0.5^{\circ} \mathrm{C} / \mathrm{min}$ & {$[6059554530]$} & $18.4 \mathrm{~min}$ \\
\hline
\end{tabular}


Table 2 Mean Square Error of Estimation (MSEE) and Prediction (MSEP) values for Single Input (1-1) and Multiple Input (5-1) PCE based Models

\begin{tabular}{|l|c|c|}
\hline & 1-1 PCE Model & 5-1 PCE Model \\
\hline Mean Square Error of Estimation & 6.33 & 0.72 \\
\hline Mean Square Error of Prediction & 11.62 & 7.05 \\
\hline
\end{tabular}




\section{Figure Captions}

Figure 1: Dropwise Additive Manufacturing System.

Figure 2: Supervisory Control Strategy for the Dropwise Additive Manufacturing Process.

Figure 3: Temperature profiles applied on the substrate.

Figure 4: Effect of different cooling profiles on the dissolution profiles of the dosage forms created a. with small drops, b. with large drops.

Figure 5: Dissolution profiles of the dosage forms created with two different drop sizes and solidified at different temperature profiles a. constant temperature, b. fast cooling, c. slow cooling.

Figure 6: PCE based surrogate model development.

Figure 7: Input-output pairs for (1-1) and (5-1) PCE-based surrogate models

Figure 8: Crystallization temperature profiles used in the PCE model development

Figure 9: Performance of $1^{\text {st }}$ order PCE model with validation and prediction for a. single input single output (1-1) b. multiple input single output (5-1) cases. Exp. data: experimental data points used in PCE model development, PCE: model validation points, Actual: the experimental time constant, which is predicted using the PCE model, Simulated: the model prediction.

Figure 10: Optimized temperature profiles using (1-1) and (5-1) PCE models vs. the actual temperature profiles

Figure 11: Hierarchical Temperature Control Strategy 


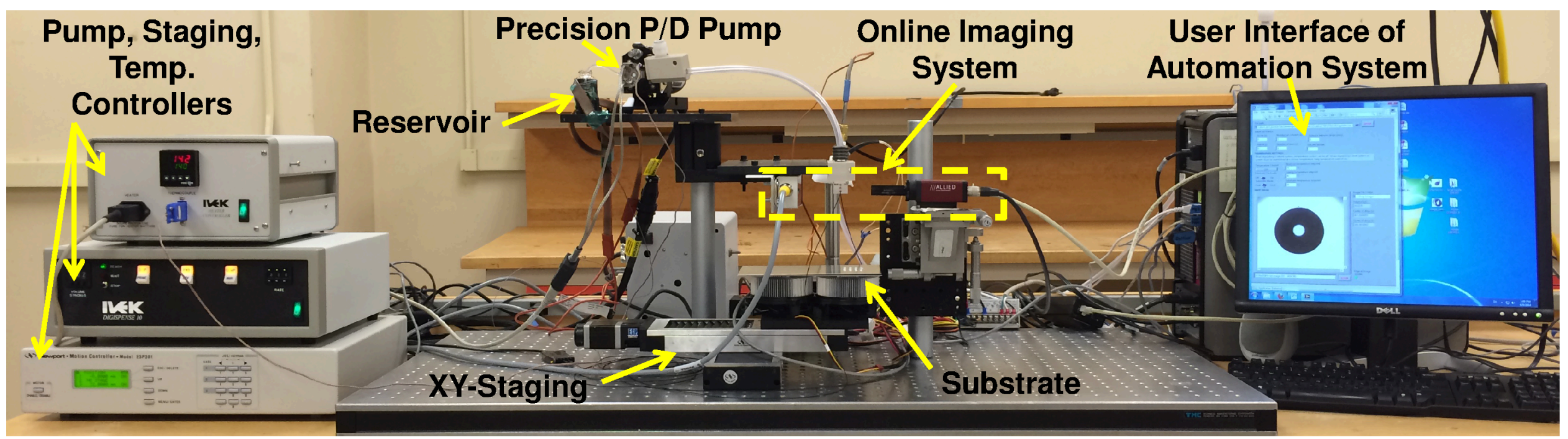




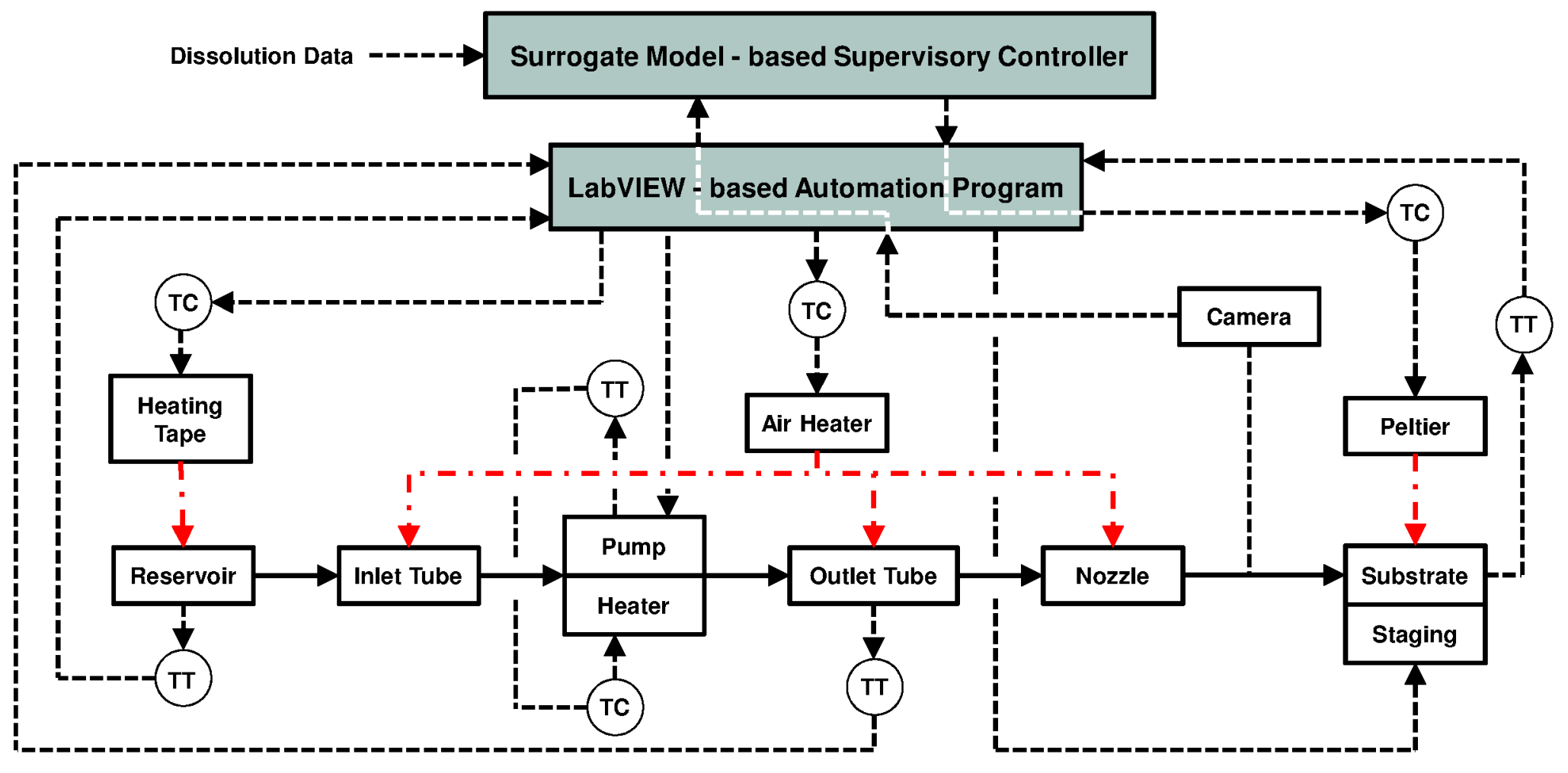




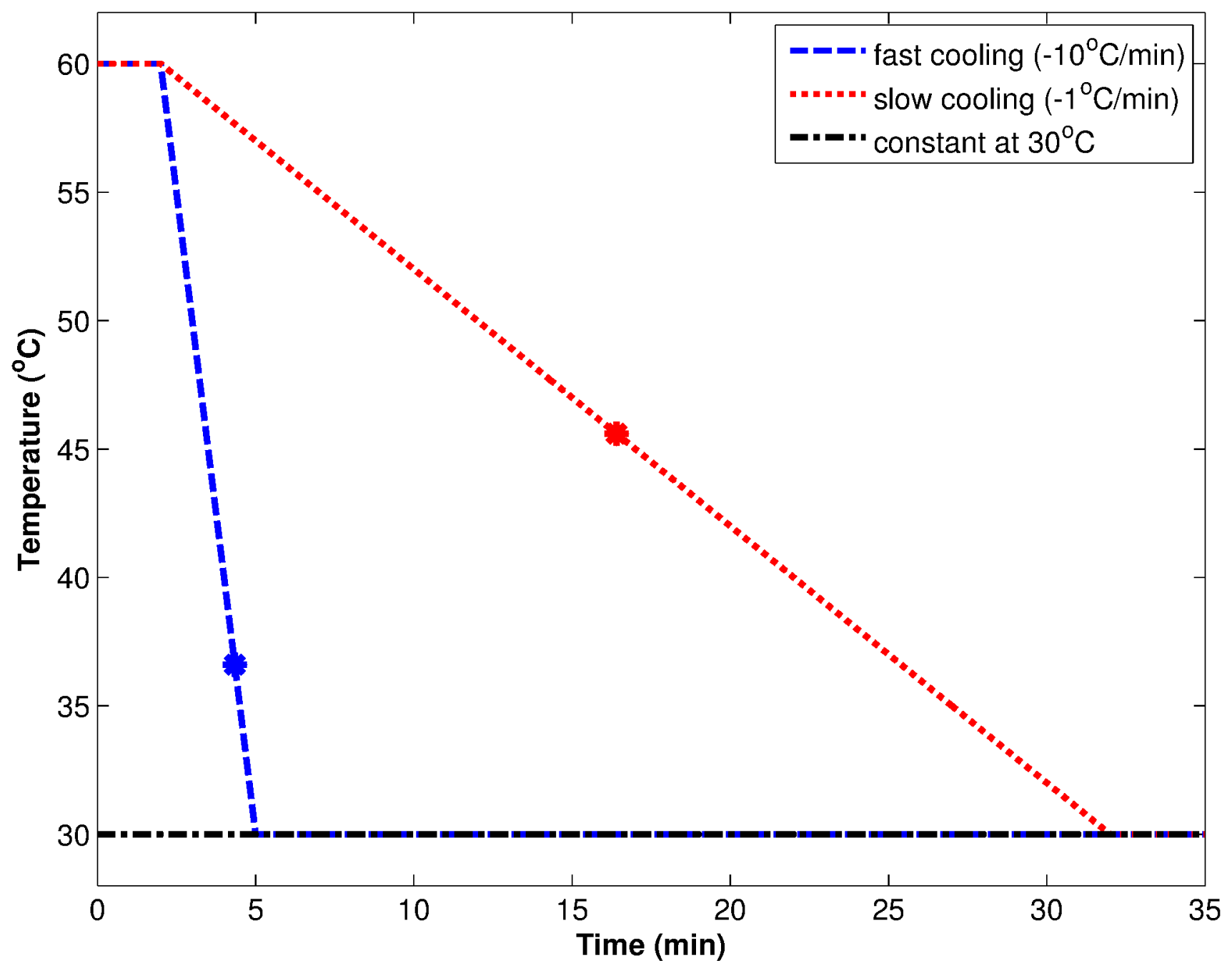




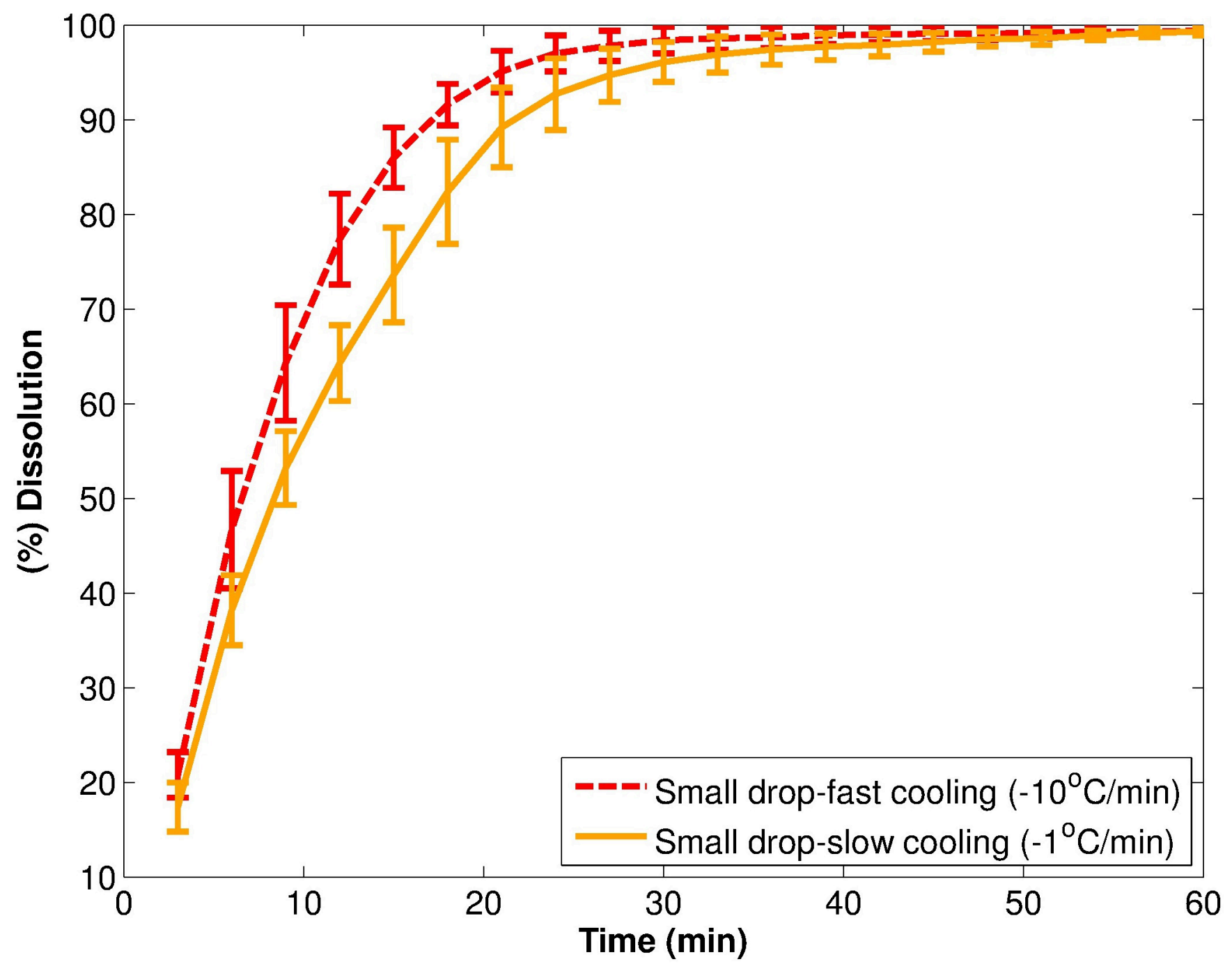




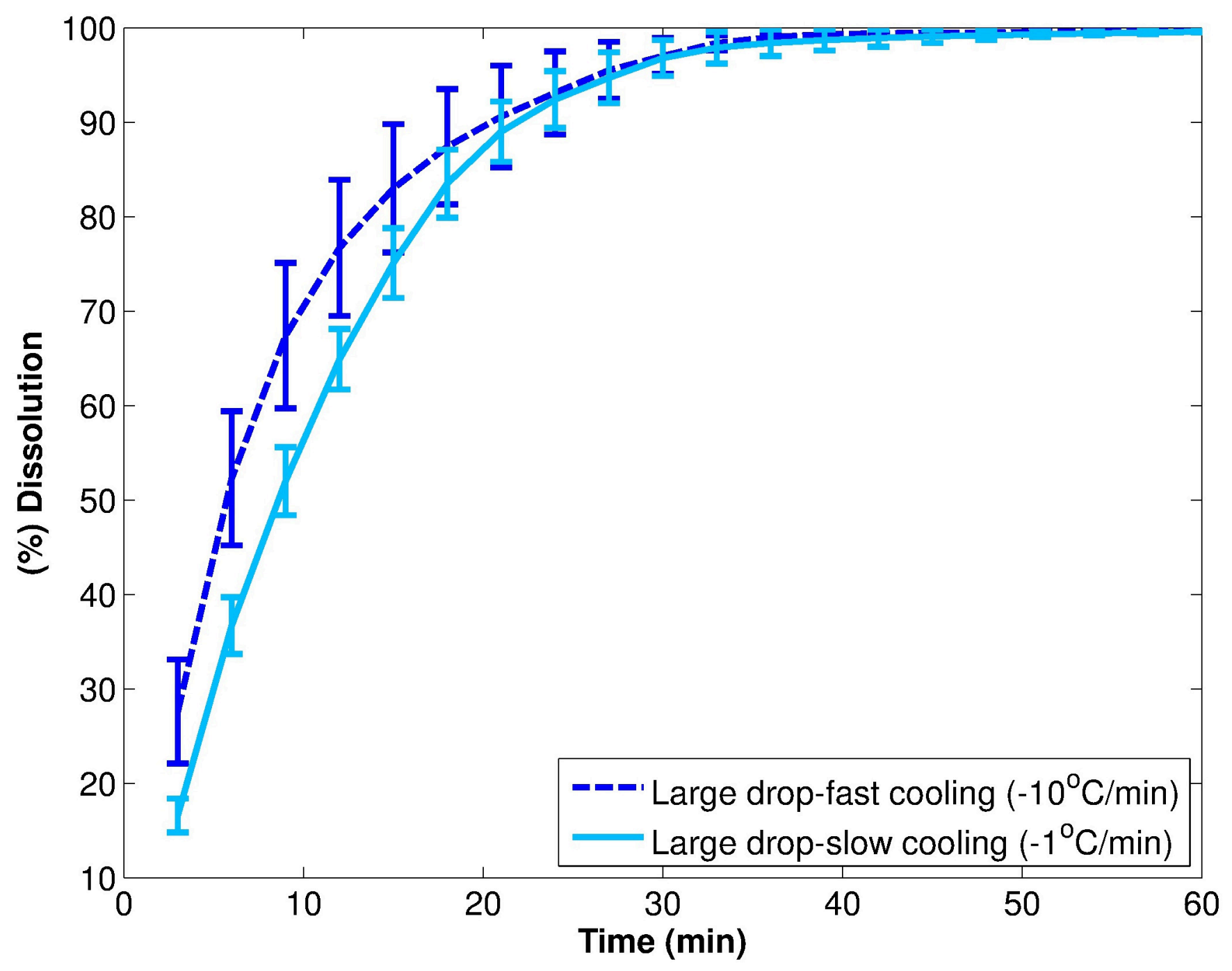




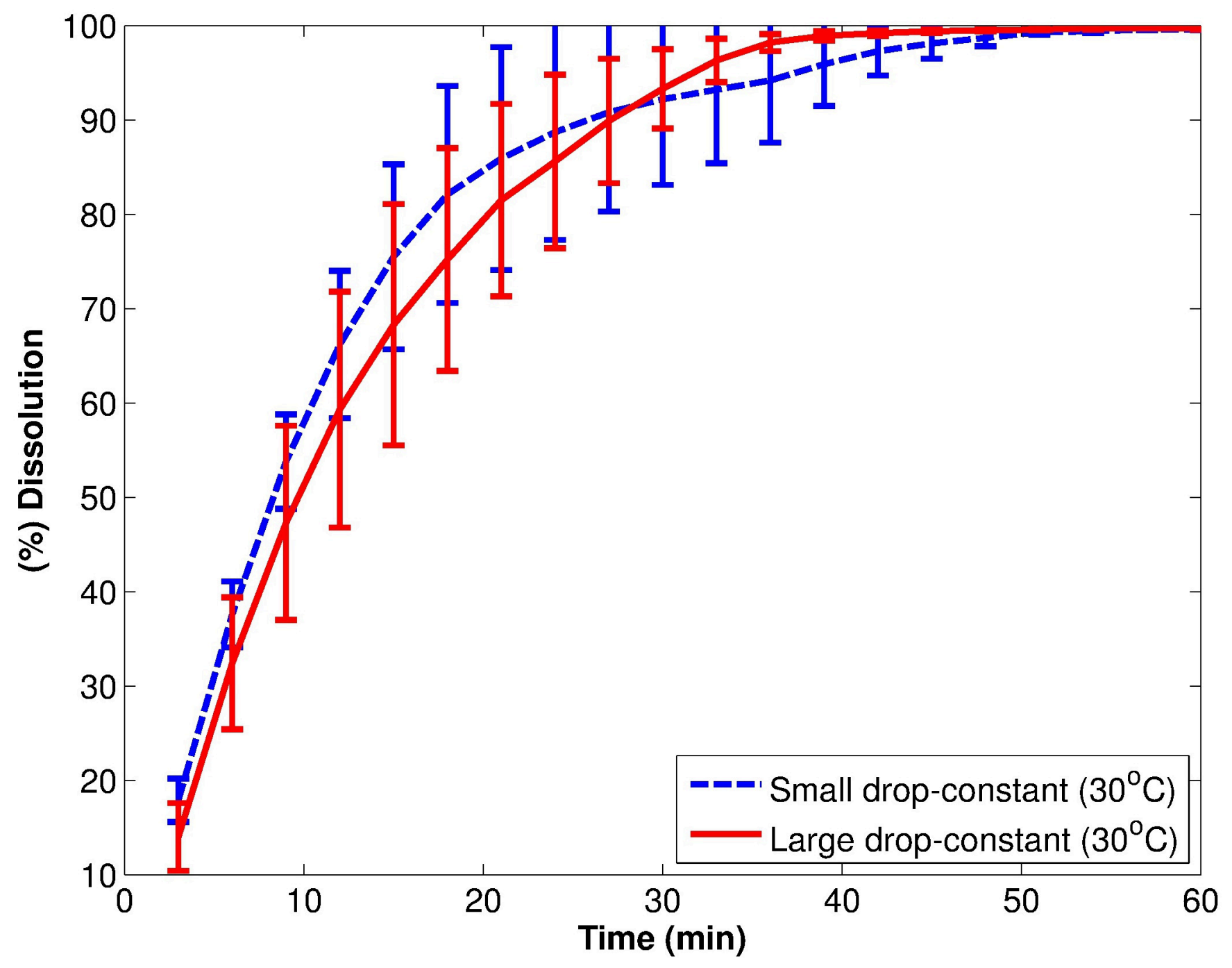




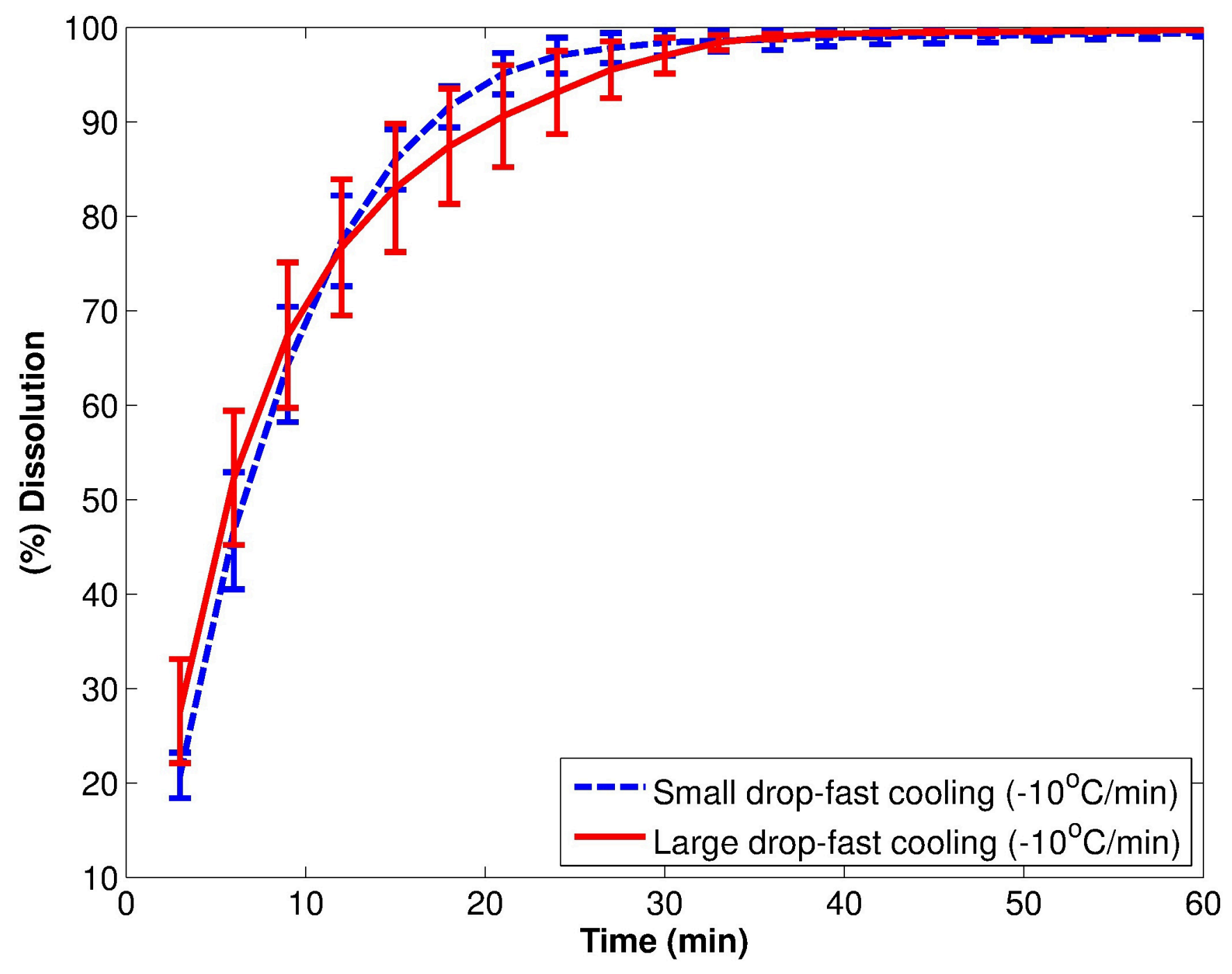




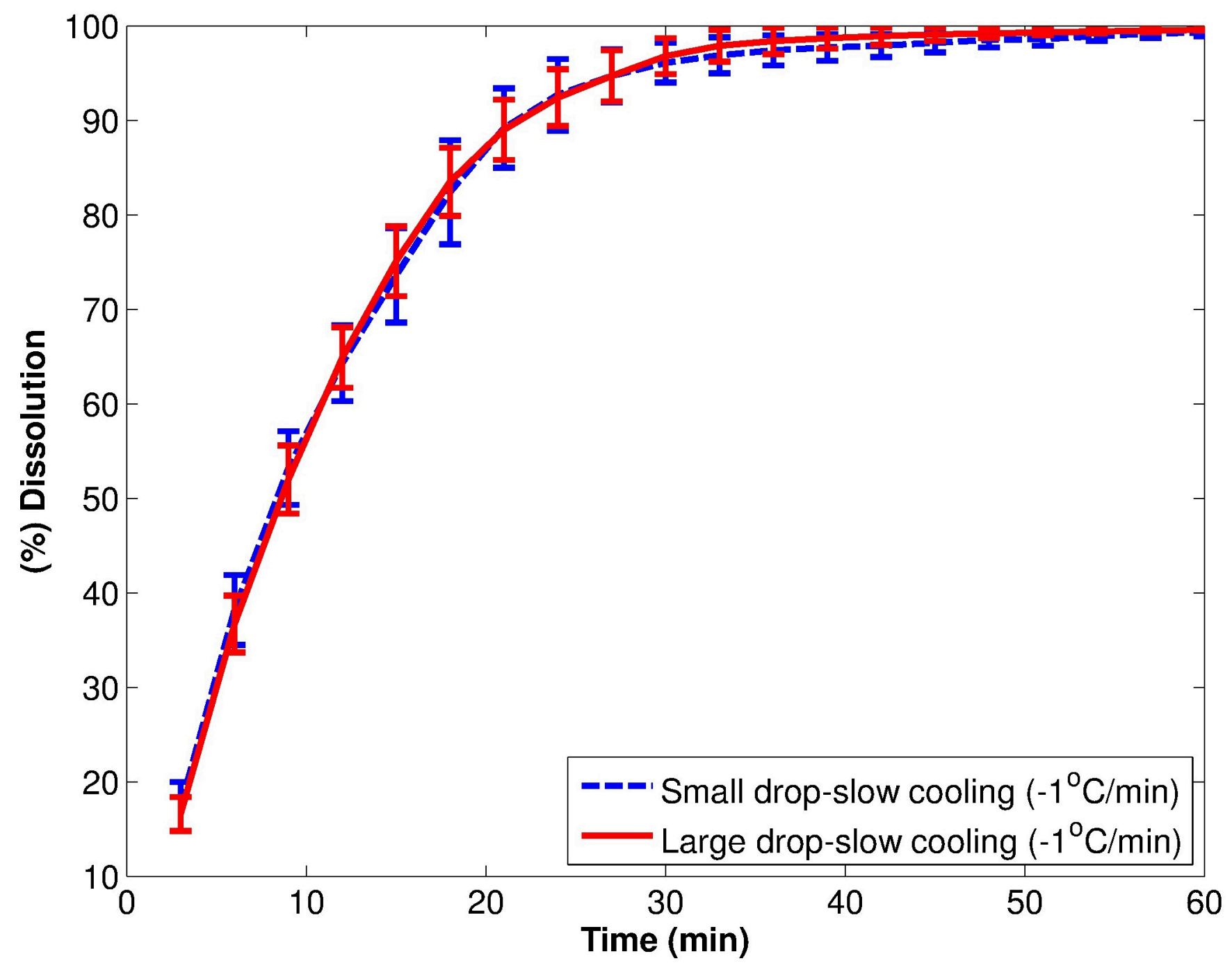


a)
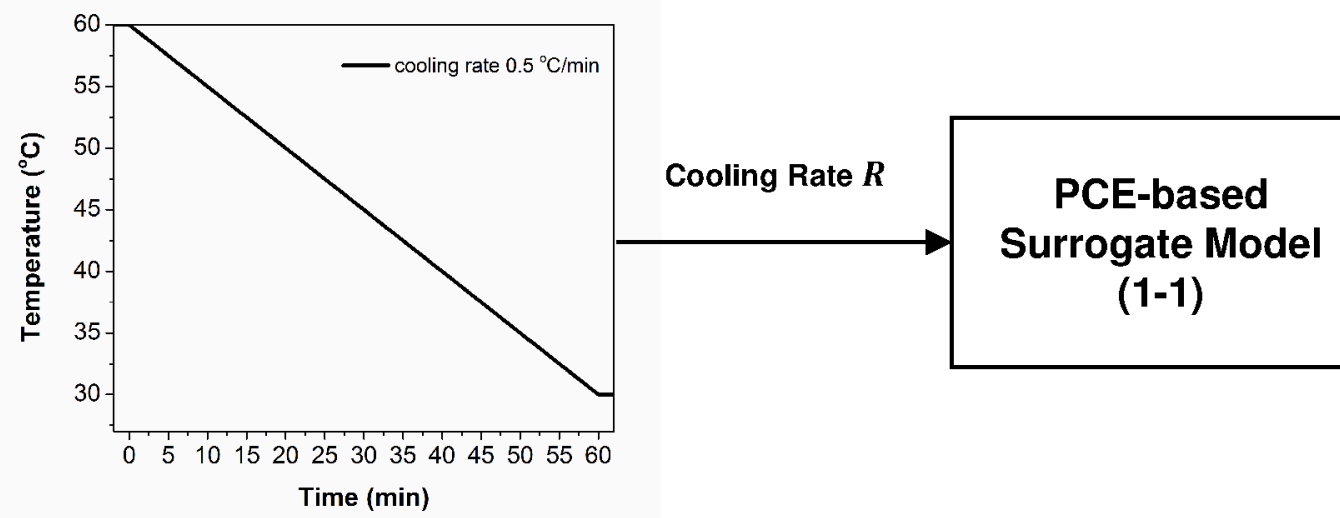

Time (min)

b)

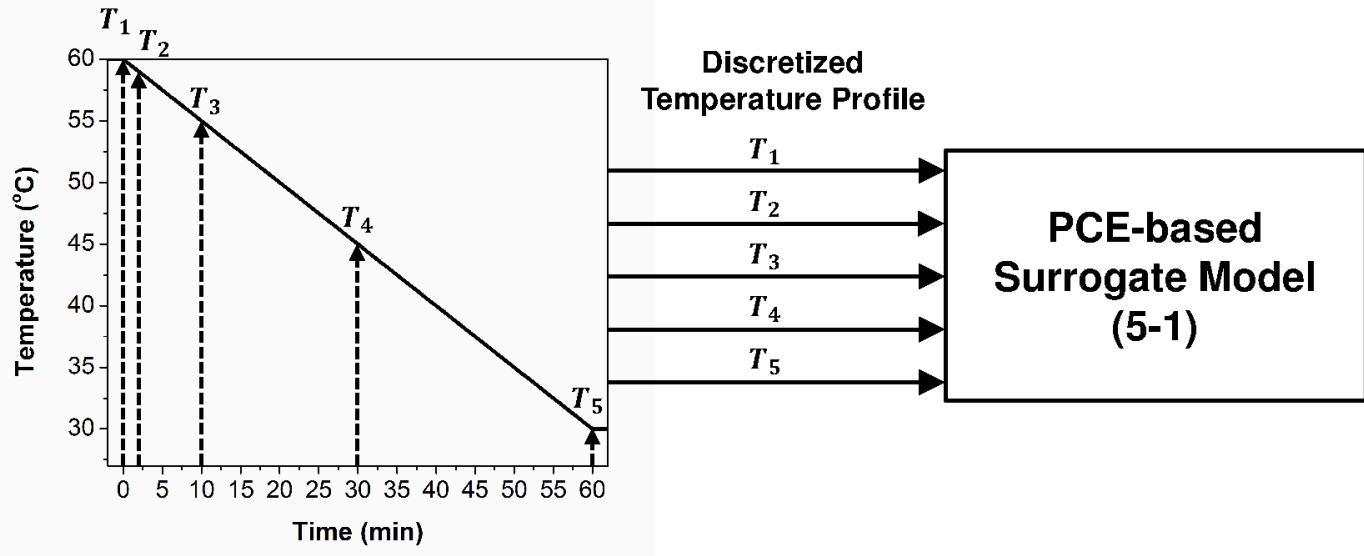

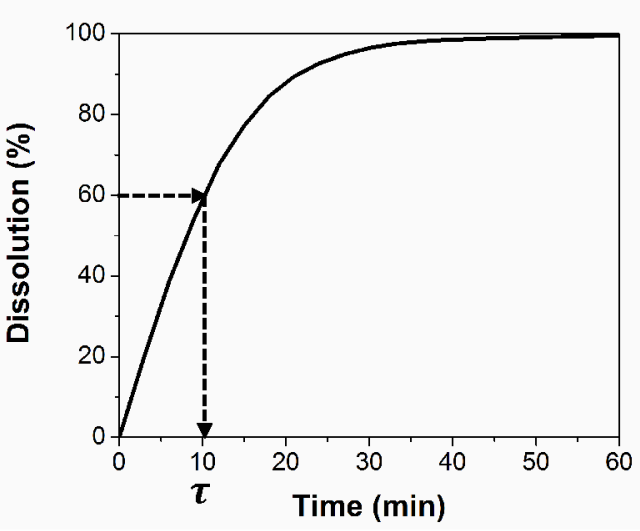

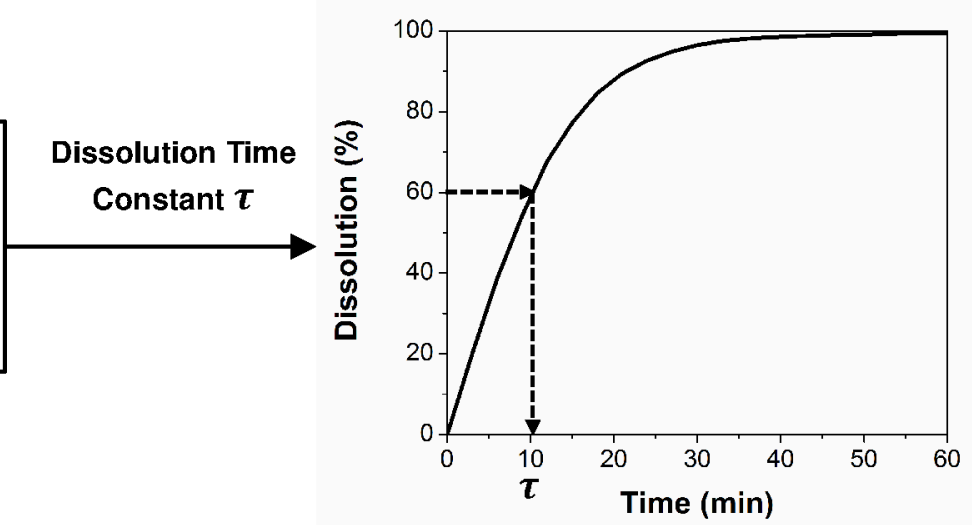




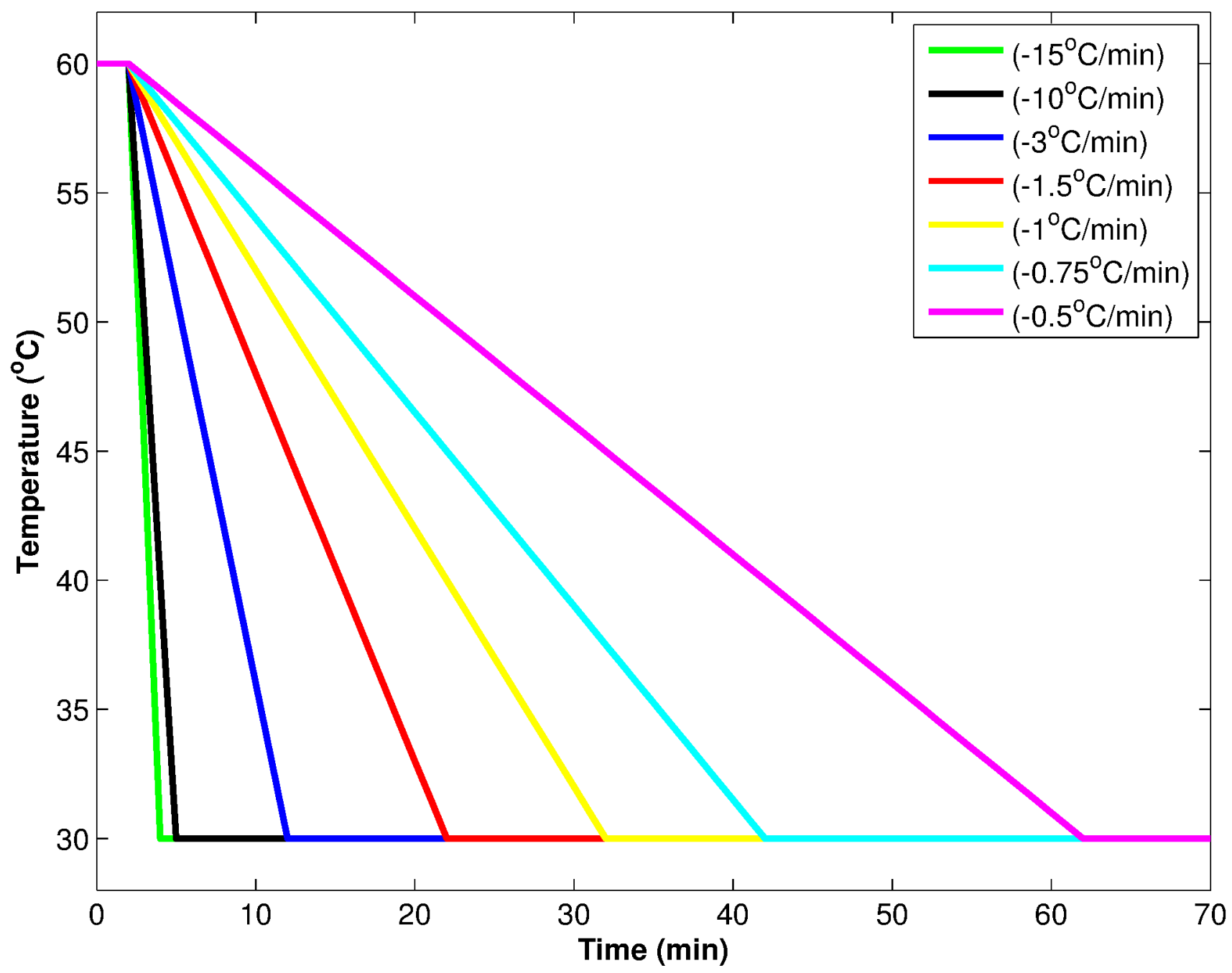



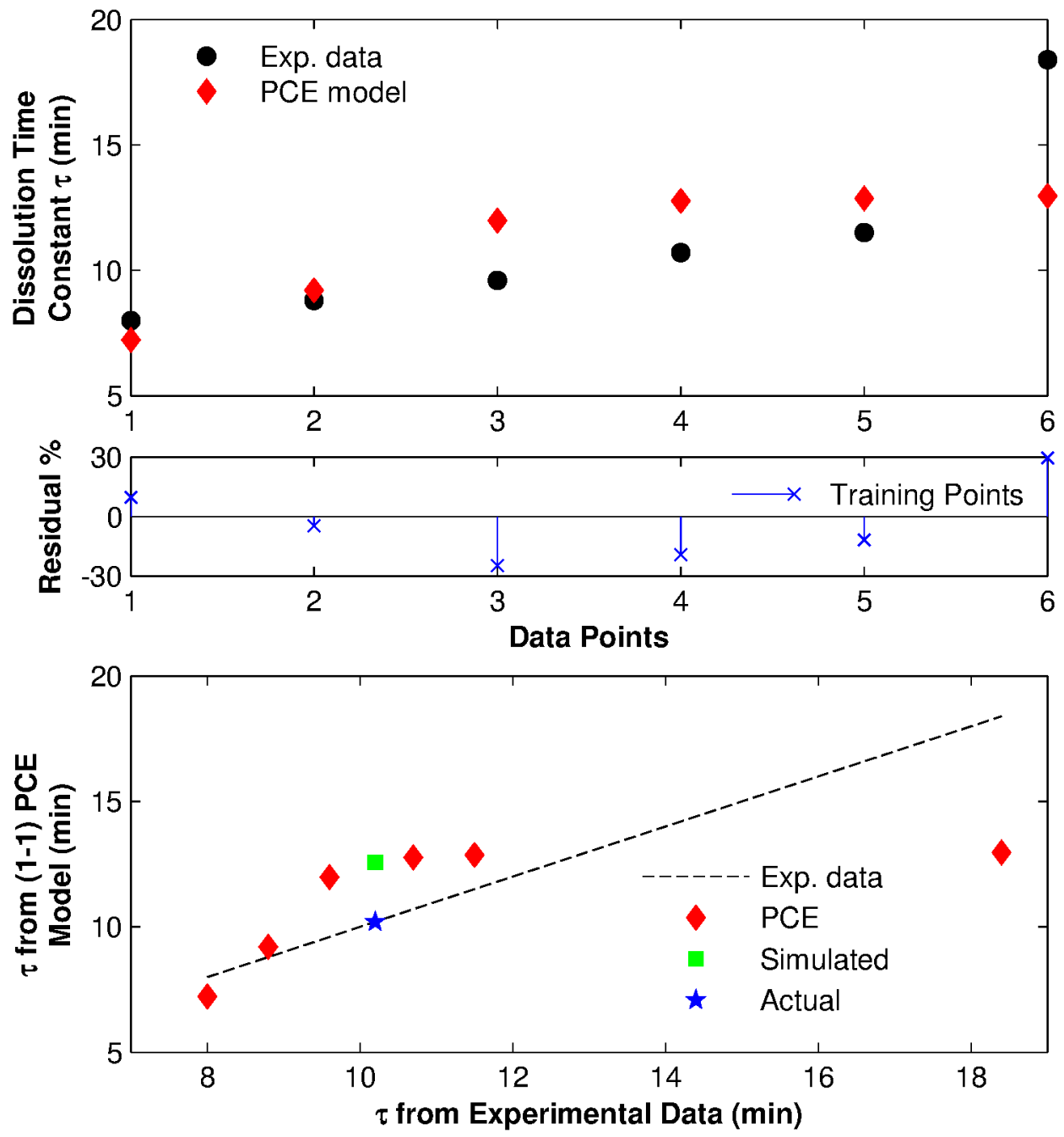

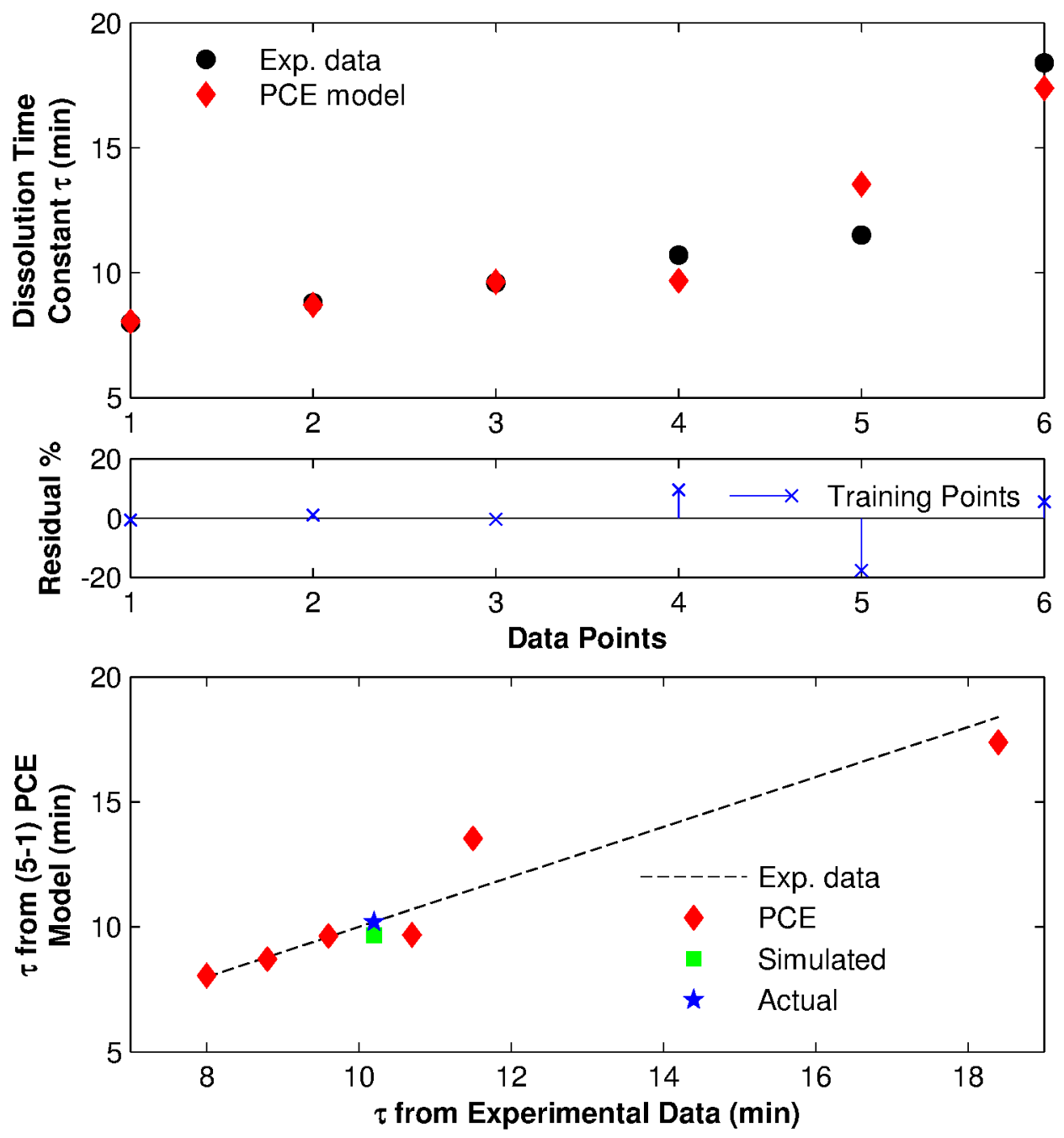

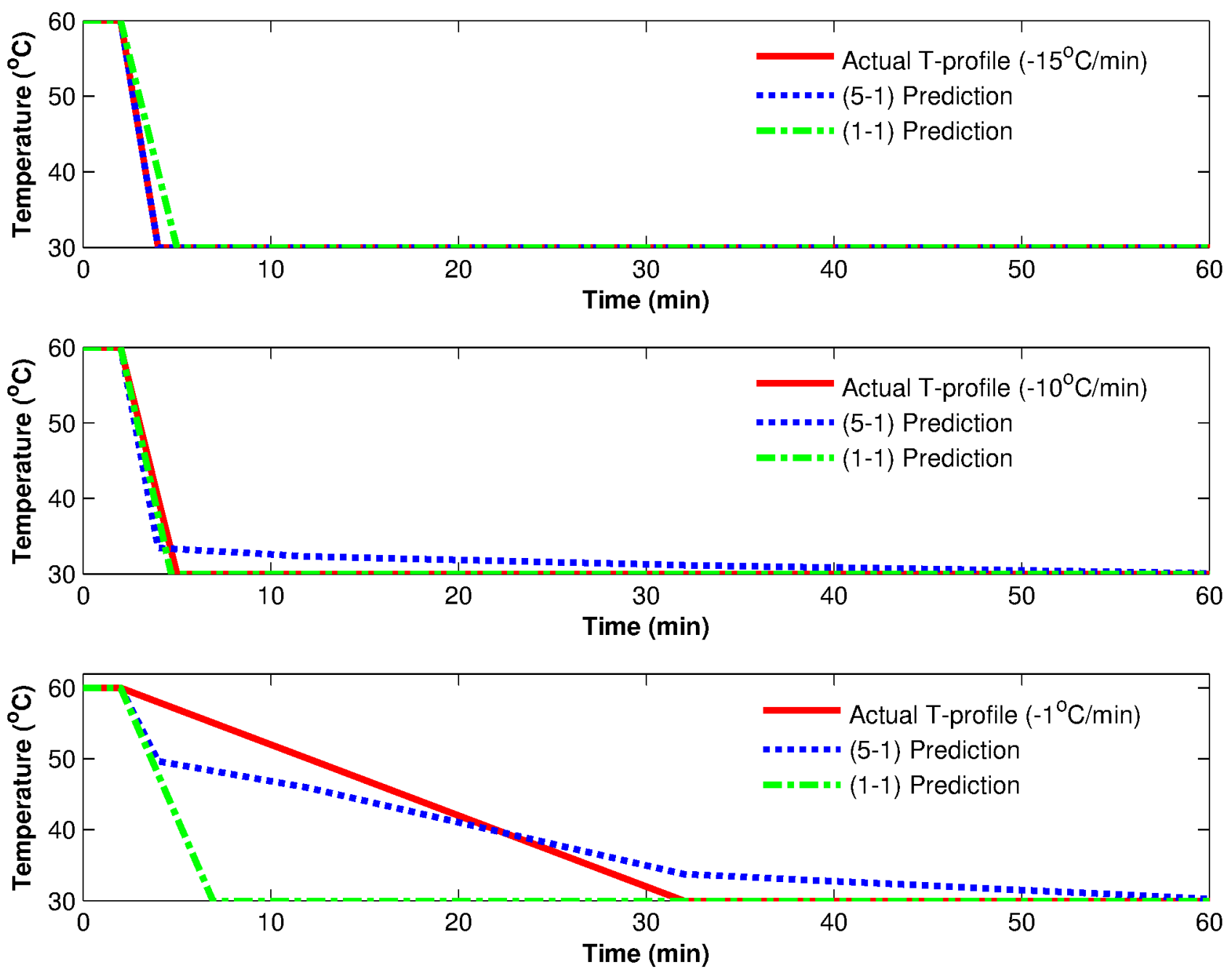


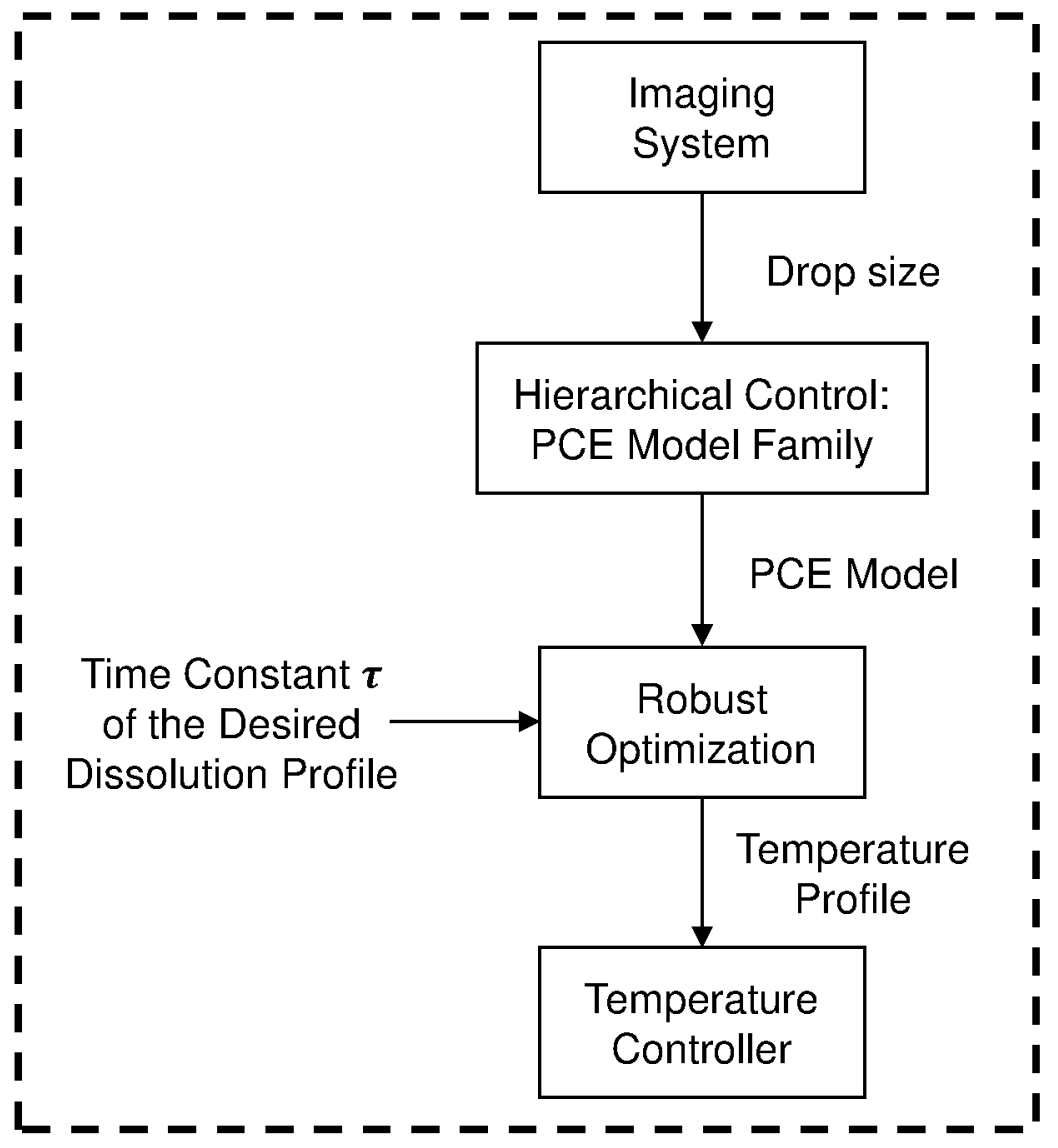

